\title{
Gamma-ray strength function and pygmy resonance in rare earth nuclei
}

\author{
A. Voinov* \\ Frank Laboratory of Neutron Physics, Joint Institute of Nuclear Research, 141980 Dubna, Moscow reg., Russia \\ M. Guttormsen, E. Melby, J. Rekstad, A. Schiller ${ }^{\dagger}$ and S. Siem \\ Department of Physics, University of Oslo, N-0316 Oslo, Norway
}

\begin{abstract}
The $\gamma$-ray strength function for $\gamma$ energies in the $1-7 \mathrm{MeV}$ region has been measured for ${ }^{161,162} \mathrm{Dy}$ and ${ }^{171,172} \mathrm{Yb}$ using the $\left({ }^{3} \mathrm{He}, \alpha \gamma\right)$ reaction. Various models are tested against the observed $\gamma$-ray strength functions. The best description is based on the Kadmenskil, Markushev and Furman E1 model with constant temperature and the Lorentzian M1 model. A $\gamma$-ray bump observed at $E_{\gamma} \sim 3 \mathrm{MeV}$ is interpreted as the so-called pygmy resonance, which has also been observed previously in $(\mathrm{n}, \gamma)$ experiments. The parameters for this resonance have been determined and compared to the available systematics.
\end{abstract}

PACS number(s): 24.30.Cz, 24.30.Gd, 25.55.Hp, 27.70.+q

\section{INTRODUCTION}

The concept of $\gamma$-ray strength functions was introduced in the fundamental work of Blatt and Weisskopf [1]. They showed that the square of the $\gamma$-transition matrix element connecting highly excited states is proportional to the level spacing $D_{i}$ of the initial states $i$ with equal spin and parity. Therefore, the ratio of the partial radiative width $\Gamma_{i}$ of the states $i$ (which is connected to $\gamma$ transitions with transition energy $E_{\gamma}$ and populating some low lying levels) and $D_{i}$ was suggested to be important for the description of $\gamma$ transitions in the continuum. The corresponding model-independent definition of the $\gamma$-ray strength function ${ }^{1}$ is given by $f_{\mathrm{X} L}=\Gamma_{i} /\left(E_{\gamma}^{2 L+1} D_{i}\right)$, where $L$ is the multipolarity of the $\gamma$ transition and $\mathrm{X}$ refers to the electric or magnetic character of the $\gamma$ transition. The $\gamma$-ray strength function is now considered as a measure for the average electromagnetic properties of nuclei and is fundamental for the understanding of nuclear structure and reactions involving $\gamma$ rays.

Experimentally, the main information on the $\gamma$-ray strength function has been obtained from the study of photoabsorption cross-sections [2]. It is commonly adopted that the E1 strength function is determined by the properties of the giant electric dipole resonance (GEDR) around its resonance energy, typically $E_{\gamma} \sim$ 10-20 MeV. However, serious lack of information persists at lower $\gamma$-ray energies. It was assumed that the tail of the Lorentzian describing the GEDR determines the E1 strength function at these energies. The only experimental data on the E1 $\gamma$-ray strength function between compound states with $\gamma$-ray energies below $2 \mathrm{MeV}$ have been obtained using the ${ }^{143} \mathrm{Nd}(\mathrm{n}, \gamma \alpha)$ reaction [3].

\footnotetext{
*email: voinov@nf.jinr.ru

${ }^{\dagger}$ Corresponding author, email: Andreas.Schiller@fys.uio.no

${ }^{1}$ In literature, also called the radiative strength function.
}

These data show that the extrapolation of the GEDR to low energies fails to describe the experimental values of the E1 strength function and indicates a finite value of $f_{\mathrm{E} 1}$ in the limit $E_{\gamma} \rightarrow 0$. As a result, a model for the E1 strength function was developed by Kadmenskiľ, Marku-

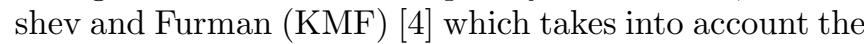
energy and temperature dependence of the GEDR width. Today, this model and its empirical modifications 50 are frequently used in the description of experimental data, but at the same time, it needs additional experimental verification.

The E1 strength function is not solely governing the $\gamma$-ray emission for lower $\gamma$-ray energies. Other multipolarities, and especially the M1 strength function, play important roles as well. The experimental information on the $\gamma$-ray strength of M1 transitions is more scarce. It is commonly assumed that the M1 strength is well described by the Weisskopf model [1], where the dipole $\gamma$-ray strength function is independent of the $\gamma$ energy. But some experiments indicate the existence of an M1 giant resonance originating from spin-flip excitations in the nucleus [6]. Also, the analysis of $\gamma$-ray spectra from $(\mathrm{n}, \gamma)$ reactions [7] indicates that the use of the M1 giant dipole resonance model gives a better fit to the experimental data than the Weisskopf model.

Special attention [8 12] has been devoted to the anomalous bump found in the $\gamma$-ray spectra of the $(\mathrm{n}, \gamma)$ and $(\mathrm{d}, \mathrm{p} \gamma)$ reactions at low energies. The same bump has probably also been observed in the $\left({ }^{3} \mathrm{He}, \alpha \gamma\right)$ reaction [13]. A previous work [8] shows that the energy of the $\gamma$-ray bump increases with neutron number in the $N=82-126$ region. The bump is called the pygmy resonance due to the considerably lower strength compared to the GEDR. The pygmy resonance has first been ex- 
plained by the enhancement of the E1 strength function [8]. However, one can not rule out a possible M1 character connected to orbital M1 strength (scissors mode) in nuclei, which was first observed in electron scattering experiments 14.

The $\gamma$-ray strength function is difficult to measure in the $\gamma$-decay between highly excited states, since the decay rate also depends on the number of accessible levels. The analysis of $\gamma$-ray strength functions from the spectra of $(\mathrm{n}, \gamma)$ reactions [7] shows that the results depend crucially on the level density model employed. Therefore, conclusions based on a certain level density formula should be considered as preliminary and thus, need confirmation.

Recently 15 17, a new experimental technique has been developed, based on a set of primary $\gamma$-ray spectra measured at consecutive excitation energies $E$ in light-ion reactions with one charged ejectile. The technique allows to disentangle the $\gamma$-ray spectra into a $\gamma$-energy dependent function $F\left(E_{\gamma}\right)$ (which, as will be shown below, can be uniquely connected to the $\gamma$-ray strength function) and the level density $\rho(E)$. It makes it possible to study the $\gamma$-ray strength function and level density independently from each other, in contrast to what can be done by using radiative neutron capture techniques. In previous works [18 20] the extracted level densities $\rho$ have been utilized to deduce thermodynamical properties for several rare earth nuclei. In this paper, however, we will focus on the $F\left(E_{\gamma}\right)$ function.

The experimental method is described in Sect. II. In Sect. III we give a short outline of the models used to describe the experimental data, and in Sect. IV we compare various predictions to the experimental data. Our results are also compared to data from the $(\mathrm{n}, \gamma)$ reaction performed by others. Conclusions are given in Sect. V.

\section{EXPERIMENTAL METHOD}

The experiments were carried out with $45 \mathrm{MeV}^{3} \mathrm{He}-$ projectiles at the Oslo Cyclotron Laboratory (OCL). The particle- $\gamma$ coincidences are measured with the CACTUS multidetector array [21] using the $\left({ }^{3} \mathrm{He}, \alpha \gamma\right)$ reaction on ${ }^{162,163} \mathrm{Dy}$ and ${ }^{172,173} \mathrm{Yb}$ self-supporting targets. The charged ejectiles were detected with eight particle telescopes placed at an angle of $45^{\circ}$ relative to the beam direction. An array of $28 \mathrm{NaI} \gamma$-ray detectors with a total efficiency of $\sim 15 \%$ of $4 \pi$ surrounded the target and particle detectors.

The experimental extraction procedure and the assumptions made are described in Refs. [15,17] and references therein. From the $\alpha-\gamma$-coincidences, spectra of the total $\gamma$-ray cascade can be sorted out according to the initial excitation energy $E$. These spectra are the basis for making the first generation (or primary) $\gamma$-ray matrix $P\left(E, E_{\gamma}\right)$, which is factorized according to the Brink-Axel hypothesis 22,23]

$$
P\left(E, E_{\gamma}\right) \propto F\left(E_{\gamma}\right) \rho\left(E-E_{\gamma}\right) .
$$

Here, $F$ and $\rho$ are the $\gamma$-ray energy dependent factor and the level density, respectively. It is now possible to determine $F$ and $\rho$ by an iterative procedure. The first trial function for $\rho$ is simply taken as a straight line and the corresponding $F$ is determined by Eq. (11). Then, a $\chi^{2}$ minimum is calculated for each data point of $F$ and $\rho$, keeping the others fixed. This procedure is repeated about 50 times, until a global least square fit to the $\sim 1400$ data points of the $P\left(E, E_{\gamma}\right)$ matrix is achieved.

It has been shown [17] that if one solution for $F$ and $\rho$ has been found, functions of the form

$$
\begin{aligned}
\tilde{\rho}\left(E-E_{\gamma}\right) & =A \rho\left(E-E_{\gamma}\right) \exp \left(\alpha\left[E-E_{\gamma}\right]\right) \\
\tilde{F}\left(E_{\gamma}\right) & =B F\left(E_{\gamma}\right) \exp \left(\alpha E_{\gamma}\right)
\end{aligned}
$$

give exactly the same fit to the $P\left(E, E_{\gamma}\right)$ matrix. The values of $A, B$ and $\alpha$ can be determined by additional conditions. The $A$ and $\alpha$ parameters are used for absolute normalization of the level density $\rho$ : They are adjusted to reproduce $(i)$ the number of levels observed in the vicinity of the ground state and (ii) the neutron resonance spacing at the neutron binding energy $B_{n}$. Further details on the extraction procedure and the simulation of errors are given in Ref. 17]. In the following we will concentrate on the $\gamma$-ray energy dependent function $F\left(E_{\gamma}\right)$ and its normalization.

We assume that the main contributions to the derived $F$ function are from E1 and M1 $\gamma$-transitions and that the accessible levels of positive and negative parity are equal in number for any energy and spin i.e.

$$
\rho\left(E-E_{\gamma}, I_{f}, \pm \Pi_{f}\right)=\frac{1}{2} \rho\left(E-E_{\gamma}, I_{f}\right) .
$$

Thus, the observed $F$ is expressed by a sum of the E1 and M1 $\gamma$-ray strength functions only

$$
B F\left(E_{\gamma}\right)=\left[f_{\mathrm{E} 1}\left(E_{\gamma}\right)+f_{\mathrm{M} 1}\left(E_{\gamma}\right)\right] E_{\gamma}^{3},
$$

where $B$ is the unknown normalization constant. Our experiment does not provide the possibility to derive the absolute normalization of $F\left(E_{\gamma}\right)$ (see Eq. (3)), therefore, the normalization constant has to be determined from other experimental data. The experimental, average total radiative width of neutron resonances $\left\langle\Gamma_{\gamma}\right\rangle$ at the neutron binding energy $B_{n}$ can e.g. be written in terms of $F$. To show this, we start with Eq. (3.1) of Ref. [5]

$$
\begin{aligned}
\left\langle\Gamma_{\gamma}(E, I, \Pi)\right\rangle= & \frac{1}{\rho(E, I, \Pi)} \sum_{\mathrm{X} L} \sum_{I_{f}, \Pi_{f}} \int_{E_{\gamma}=0}^{E} \mathrm{~d} E_{\gamma} \\
& E_{\gamma}^{2 L+1} f_{\mathrm{X} L}\left(E_{\gamma}\right) \rho\left(E-E_{\gamma}, I_{f}, \Pi_{f}\right)
\end{aligned}
$$

where $\left\langle\Gamma_{\gamma}(E, I, \Pi)\right\rangle$ is the average total radiative width of levels with energy $E$, spin $I$ and parity $\Pi$. The summations and integration are going over all final levels with spin $I_{f}$ and parity $\Pi_{f}$ which are accessible by $\gamma$ radiation with energy $E_{\gamma}$, multipolarity $L$ and electromagnetic 
character X. If we, again, assume that only dipole radiation contributes significantly to the sum and that the number of accessible levels with positive and negative parity are equal, we obtain, by combining Eqs. (5) and (6), the average total radiative width of neutron s-wave capture resonances with spins $I_{t} \pm 1 / 2$ expressed in terms of the $F\left(E_{\gamma}\right)$ function

$$
\begin{aligned}
& \left\langle\Gamma_{\gamma}\left(B_{n}, I_{t} \pm 1 / 2, \Pi_{t}\right)\right\rangle=\frac{1}{2 \rho\left(B_{n}, I_{t} \pm 1 / 2, \Pi_{t}\right)} \int_{E_{\gamma}=0}^{B_{n}} \mathrm{~d} E_{\gamma} \\
& B F\left(E_{\gamma}\right) \rho\left(B_{n}-E_{\gamma}\right) \sum_{J=-1}^{1} g\left(B_{n}-E_{\gamma}, I_{t} \pm 1 / 2+J\right), \quad(7)
\end{aligned}
$$

where $I_{t}$ and $\Pi_{t}$ are the spin and parity of the target nucleus in the $(\mathrm{n}, \gamma)$ reaction and $\rho$ is the experimental level density. Furthermore, we have expressed $\rho$ as the product of the total level density, summed over all spins and the spin distribution $g$. The spin distribution of the level density is given by 24

$$
g(E, I)=\frac{2 I+1}{2 \sigma^{2}} \exp \left[-(I+1 / 2)^{2} / 2 \sigma^{2}\right],
$$

where $\sigma$ is the excitation-energy dependent spin cutoff parameter. The spin distribution is normalized to $\sum_{I} g \approx 1$. The experimental value of the average total radiative width of neutron resonances $\left\langle\Gamma_{\gamma}\right\rangle$ is then the weighted sum of contributions with $I_{t} \pm 1 / 2$ according to Eq. (7).

Because of methodical difficulties, the functions $F\left(E_{\gamma}\right)$ and $\rho(E)$ can not be determined experimentally in the interval $E_{\gamma}<1 \mathrm{MeV}$ and $E>B_{n}-1 \mathrm{MeV}$, respectively. In addition, the data at the highest $\gamma$-ray energies, $E_{\gamma}>B_{n}-1 \mathrm{MeV}$, suffer from poor statistics. Therefore, extrapolations of $F$ and $\rho$ were necessary in order to calculate the integral in Eq. (7). The contribution from the extrapolation to the total radiative width in Eq. (7) does not exceed $15 \%$, thus the errors due to a possibly poor extrapolation are expected to be of minor importance.

\section{MODELS FOR E1 AND M1 RADIATION}

There have been developed several models for the $\gamma$-ray strength functions $f_{\mathrm{X} L}$. The theories behind the models are complicated, and will not be outlined here. However, the resulting strength functions can be written in simple analytical forms. In this work, we have tested various E1 and M1 models. For E1 $\gamma$-transitions these are:

- The standard giant electric dipole resonance (GEDR) model based on the Brink-Axel approach 22,23

\footnotetext{
${ }^{2}$ The constant $1 /\left(3 \pi^{2} \hbar^{2} c^{2}\right)$ equals $8.6 \times 10^{-8} \mathrm{mb}^{-1} \mathrm{MeV}^{-2}$.
}

$$
f_{\mathrm{E} 1}\left(E_{\gamma}\right)=\frac{1}{3 \pi^{2} \hbar^{2} c^{2}} \frac{\sigma_{\mathrm{E} 1} E_{\gamma} \Gamma_{\mathrm{E} 1}^{2}}{\left(E_{\gamma}^{2}-E_{\mathrm{E} 1}^{2}\right)^{2}+E_{\gamma}^{2} \Gamma_{\mathrm{E} 1}^{2}}
$$

where $\sigma_{\mathrm{E} 1}, \Gamma_{\mathrm{E} 1}$ and $E_{\mathrm{E} 1}$ are the giant electric dipole resonance parameters derived from photoabsorption experiments. ${ }^{2}$

- The model of Kadmenskiı, Markushev and Furman

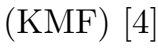

$$
f_{\mathrm{E} 1}\left(E_{\gamma}\right)=\frac{1}{3 \pi^{2} \hbar^{2} c^{2}} \frac{0.7 \sigma_{\mathrm{E} 1} \Gamma_{\mathrm{E} 1}^{2}\left(E_{\gamma}^{2}+4 \pi^{2} T^{2}\right)}{E_{\mathrm{E} 1}\left(E_{\gamma}^{2}-E_{\mathrm{E} 1}^{2}\right)^{2}},
$$

where $T$ is the temperature of the nucleus which is usually determined as $T=\sqrt{U / a}$ with $U$ being the shifted excitation energy and $a$ the level density parameter. The energy and temperature dependent width of the GEDR in this model is expressed by

$$
\Gamma_{\mathrm{E} 1}\left(E_{\gamma}, T\right)=\frac{\Gamma_{\mathrm{E} 1}}{E_{\mathrm{E} 1}^{2}}\left(E_{\gamma}^{2}+4 \pi^{2} T^{2}\right) .
$$

These expressions are developed in the framework of a collisional damping model for $E_{\gamma}<E_{\mathrm{E} 1}$ and although they should hold for $T \ll 2 \mathrm{MeV}$, the absence of thermal shape fluctuations in the model limits their validity to $T<1 \mathrm{MeV}$.

For deformed nuclei, the giant dipole resonance is split into two components, hence the sum of two strength functions with different GEDR parameters has been employed.

For M1 $\gamma$-transitions we are testing:

- The adjusted single-particle model of Weisskopf 11 where $f_{\mathrm{M} 1}\left(E_{\gamma}\right)$ is independent of $E_{\gamma}$ and the absolute value of $f_{\mathrm{M} 1}$ has been taken from $f_{\mathrm{M} 1} / f_{\mathrm{E} 1}$ systematics close to the neutron binding energy [25].

- A Lorentzian based on the existence of a giant magnetic dipole resonance (GMDR) which is assumed to be related to the spin-flip transition between single-particle states [6]. The $\gamma$-ray strength function in this case is determined by

$$
f_{\mathrm{M} 1}\left(E_{\gamma}\right)=\frac{1}{3 \pi^{2} \hbar^{2} c^{2}} \frac{\sigma_{\mathrm{M} 1} E_{\gamma} \Gamma_{\mathrm{M} 1}^{2}}{\left(E_{\gamma}^{2}-E_{\mathrm{M} 1}^{2}\right)^{2}+E_{\gamma}^{2} \Gamma_{\mathrm{M} 1}^{2}} .
$$

In the following, we will compare these models to the experimental findings. 


\section{RESULTS AND DISCUSSION}

Figure 1 shows the experimentally extracted $\gamma$-ray energy dependent factor $F$ and the level density $\rho$ for ${ }^{161,162} \mathrm{Dy}$ and ${ }^{171,172} \mathrm{Yb}$. These data are the very same as in Ref. 19], except that the data of ${ }^{171} \mathrm{Yb}$ have been retuned by adjusting the parameters $A$ and $\alpha$ to fit the level density based on known discrete levels at low excitation energy. We recognize that the shape of the unnormalized $F$ functions are rather equal for neighboring isotopes indicating that $F$ is a slowly varying function of mass number.

In the extraction procedure, we have used $\gamma$-ray spectra from excitation energy bins between 4 and $8 \mathrm{MeV}$. This span in excitation energy corresponds to a temperature region of 0.5 to $0.7 \mathrm{MeV}$ for the initial states, and 0.4 to $0.6 \mathrm{MeV}$ for the final states. The spin window in the pick-up reaction is $2-6 \hbar$, which is assumed to be approximately equal for initial and final states. Hence, the discussion below concerns average properties of nuclei for $T \sim 0.5 \mathrm{MeV}$ and $I \sim 4 \hbar$ with the assumption of equal density of positive and negative parity states.

The normalized experimental $\gamma$-ray strength functions $f=B F / E_{\gamma}^{3}$ for ${ }^{161,162} \mathrm{Dy}$ and ${ }^{171,172} \mathrm{Yb}$ are presented in Fig. 2. The experimental values of the average total radiative width $\left\langle\Gamma_{\gamma}\right\rangle$ [26] used to determine the normal-

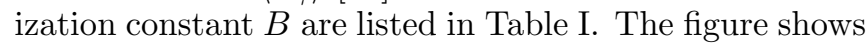
that each experimental curve consists of two components. The first one is a smooth function of the $\gamma$-ray energy and the second one is connected to a local enhancement of the $\gamma$-ray strength function at low $\gamma$-ray energies $(\sim 3 \mathrm{MeV})$. The latter component is due to the pygmy resonance, which was first observed in $(n, \gamma)$ reactions 8 .

Theoretical curves calculated with the models of Sect. III are shown as dashed curves in Fig. 2. The parameters adopted in the description of the GEDR and GMDR are presented in Table I. The GEDR parameters have been determined from the interpolation of systematics over neighboring isotopes [2,27]. For the GMDR parameters, there are no rich experimental systematics available. Our parameters have been taken from Ref. [5], namely $E_{\mathrm{M} 1}=41 A^{-1 / 3} \mathrm{MeV}$ and $\Gamma_{\mathrm{M} 1}=4 \mathrm{MeV}$. The value of $\sigma_{\mathrm{M} 1}$ has been derived from $f_{\mathrm{E} 1} / f_{\mathrm{M} 1}$ systematics at $\gamma$-ray energies close to the neutron binding energy [25].

Figure 2 also shows that the Lorentzian E1 model [label a), Eq. (9)] gives an acceptable description for $\gamma$-ray energies near $B_{n}$, in accordance with the systematics of Kopecky and Uhl for deformed nuclei [25]. But for lower energies this GEDR model overestimates the experimental data. The combination of the KMF E1 model and the Weisskopf M1 model [label b)] fails to describe the data due to the strong M1 component especially in the region of low $\gamma$-ray energies. The KMF model plus the Lorentzian M1 model [label c), Eqs. (10, 12)] is seen to give the best description of the general slope of the $\gamma$ ray strength function. However, since the M1 strengthfunction model is generally only $\sim 20 \%$ of the GEDR model for the investigated nuclei [25], no further conclusion concerning M1 models could be drawn in this work. In detail, the agreement of the last curve with the data for 161,162 Dy is satisfactory, excluding the low energy region where the pygmy resonance is observed. For ${ }^{171,172} \mathrm{Yb}$, the slopes of the calculated curves differ somewhat from the experimental ones.

In oder to obtain a good parameterization of the $\gamma$-ray strength function which can fit the experimental data, the sum of the KMF E1 and the Lorentzian M1 models has been selected for further modification. In contrast to the common use, where the nuclear temperature is defined as $T=\sqrt{U / a}$, we will keep the temperature fixed (as first proposed by Grudzevich [28,29]) according to a constant temperature model of the nuclear level density, which is supported by recent findings [19]30. The constant temperature model may also be regarded as to mimic the generalized superfluid model of the nuclear level density [31,32] in this excitation energy region. The mean value of the temperature in the excitation energy region under study is $T \sim 0.5 \mathrm{MeV}$ for the final levels as has been mentioned above. We should point out that the temperature dependence of the GEDR width used in the E1 model is a much disputed topic. Experimental data on damping of the GEDR at low temperatures $(T<1 \mathrm{MeV})$ are absent. At higher temperatures, the damping of the GEDR is intensively studied with inelastic scattering of light particles (e.g. $\alpha$ particles [33]) but different theoretical approaches give ambiguous results. For example, in the adiabatic coupling model [34,35] the increasing width is explained in terms of thermally induced shape fluctuations, yielding in general a $\Gamma \propto \sqrt{T}$ dependence. These shape fluctuations become important for $T>1-2 \mathrm{MeV}$. In the collisional damping model [36] the width of the GEDR is due to collisional damping of nucleons, giving a $\Gamma \propto T^{2}$ law. Experiments on ${ }^{208} \mathrm{~Pb}$ show that the data set can be fitted by both of these parameterizations [33], while new calculations on the collisional damping model using realistic in-medium cross-sections [36] show that the width is in general underestimated within this model. Also, a recent calculation 37 on ${ }^{120} \mathrm{Sn}$ within the phonon damping model shows good agreement with experiment.

At temperatures appropriate for the present study ( $T \sim 0.5 \mathrm{MeV})$, pairing correlations [37] and shell effects 34,35 have to be taken into account. Most experimental data on the strength function at the low-energetic tale of the GEDR are obtained from $(n, \gamma)$ reactions, where the quadratic temperature-dependence of the GEDR width [4] is a popular parameterization [5, 4, 38]. We therefore use this parameterization, knowing that the model behind can not account properly for the damping mechanism of the GEDR 36].

In order to obtain a good fit of the chosen $\gamma$-ray strength-function models to the data we use the temperature as a free parameter because of the uncertain temperature dependence of the GEDR width in our temperature region. Also, a common normalization constant 
$K$ was introduced as a free parameter. Additionally, in order to fit the experimental data in the low-energy region, a Lorentzian

$$
f_{\mathrm{py}}\left(E_{\gamma}\right)=\frac{1}{3 \pi^{2} \hbar^{2} c^{2}} \frac{\sigma_{\mathrm{py}} E_{\gamma} \Gamma_{\mathrm{py}}^{2}}{\left(E_{\gamma}^{2}-E_{\mathrm{py}}^{2}\right)^{2}+E_{\gamma}^{2} \Gamma_{\mathrm{py}}^{2}}
$$

with three free parameters $\sigma_{\mathrm{py}}, \Gamma_{\mathrm{py}}$ and $E_{\mathrm{py}}$ has been used for describing the pygmy resonance. Hence, the fitting function consists of the sum

$$
f=K\left(f_{\mathrm{E} 1}+f_{\mathrm{M} 1}\right)+f_{\mathrm{py}}
$$

where $f_{\mathrm{E} 1}$ and $f_{\mathrm{M} 1}$ are given by Eqs. (10) and (12) with parameters from systematics (see Table 1 ). The values of the fitting parameters for the pygmy resonance, the normalization constant $K$ and the temperature $T$ are quoted in Table II. The resulting curves are shown as solid lines in Fig. 2. The agreement with experiment is excellent. It can of course be debated that other models for the $\gamma$ ray strength functions can be fitted to the experimental data by letting a sufficient number of parameters free for fitting. We have chosen the sum of the KMF E1 and the Lorentzian M1 models with one fit parameter $(T)$ and one free overall normalization constant $(K)$ in order to obtain a simple parameterization of the experimental data for further applications. In addition this parameterization is convenient to extract the parameters of the pygmy resonance experimentally.

To judge the relevance of the adopted model of Eq. (14), it is important to discuss the fitting values obtained. Table II shows that the coefficient $K$ is close to 1.0 for all four nuclei ${ }^{3}$. This means that the adopted model reproduces the absolute values of the $\gamma$-ray strength functions. The small deviation of $K$ from 1.0 can be explained e.g. by uncertain values of the GEDR parameters for the investigated nuclei, or they can be ascribed uncertainties in the experimental normalization of the $\gamma$-ray strength functions.

A close inspection of the fitting of Eq. (14) to the data shows that the parameter $T$ is mainly determined by the experimental value of the $\gamma$-ray strength function in a small $\gamma$-energy region around $1.5 \mathrm{MeV}$. The fitted values for the nuclear temperature are found around $T=0.3$ $\mathrm{MeV}$, a value which is consistent for all four nuclei within the fitting errors. However, the value is lower than expected from other studies 18,19, giving $T \sim 0.5 \mathrm{MeV}$. Since the temperature dependence of the GEDR is only roughly determined by theory, we therefore interpret $T$ as a fit parameter not necessarily equal to the nuclear temperature.

It is interesting to compare the pygmy resonance parameters to those obtained from $(n, \gamma)$ experiments. Un-

\footnotetext{
${ }^{3}$ Here, the phrase "close to" should be appropriate, since the normalization in other works is often a factor of 2 uncertain.
}

fortunately, the available systematics on pygmy resonances is very scarce [8] and it is difficult to make definite conclusions. Nevertheless, Fig. 3 shows that our fitted pygmy resonance parameters are in good agreement with Ref. [8]. Both the resonance energy and the width of the pygmy resonances fit into the systematics. Our strength parameter $\sigma_{\mathrm{py}}$ is in between the values $\sigma_{\mathrm{pv}}$ and $k \sigma_{\text {py }}$ found in the $(\mathrm{n}, \gamma)$ experiment [8], see Fig. 3. The comparison supports that the pygmy resonance observed in the two reactions has the same physical origin and manifests itself as a common property of the $\gamma$-ray strength function.

As a last test, we have calculated a $\gamma$-ray spectrum using the extracted $\gamma$-ray strength function $f$ and level density $\rho$ for ${ }^{162}$ Dy. The spectrum includes $\gamma$-ray cascades from an excitation energy of $E=B_{n}+E_{n}$ and down to the ground state. The spectrum is calculated using a Monte Carlo simulation, where $f$ and $\rho$ determine the decay pattern. In Fig. A this spectrum is compared to the $\gamma$-ray spectrum from the ${ }^{161} \mathrm{Dy}(\mathrm{n}, \gamma){ }^{162} \mathrm{Dy}$ reaction (data points with error bars) measured at the neutron energy $E_{n}=47 \mathrm{keV}$ [39]. Since the $\left({ }^{3} \mathrm{He}, \alpha \gamma\right)$ data are based on a broader spin window, details in our spectra are expected to be more smeared out at final excitation energies having low level density. This concerns the fine structures seen below $2 \mathrm{MeV}$ and above $5 \mathrm{MeV}$ of $\gamma$-ray energy. The overall agreement between the two spectra is gratifying and supports our results.

\section{CONCLUSIONS}

The $\gamma$-ray energy dependent factor and the level density for ${ }^{161,162} \mathrm{Dy}$ and ${ }^{171,172} \mathrm{Yb}$ have been measured using the $\left({ }^{3} \mathrm{He}, \alpha \gamma\right)$ reaction. For the first time, the normalized $\gamma$-ray strength function $f\left(E_{\gamma}\right)$ could be extracted from such data.

Various models are tested against the observed $\gamma$-ray strength function and the best description is found for the E1 model of KMF with a fixed temperature plus Lorentzian models for the GMDR and the pygmy resonance. The pygmy resonance parameters for ${ }^{161,162} \mathrm{Dy}$ and ${ }^{171,172} \mathrm{Yb}$ fit into the available systematics obtained from $(n, \gamma)$ experiments. Hence, the adopted approach gives consistent $\gamma$-ray strength functions for the investigated nuclei.

A few tentative explanations exist for the pygmy resonance. Still, the question remains open whether the pygmy resonance is of E1 or M1 character. Measurements of the electromagnetic character of the pygmy resonance is therefore important in order to pin down the true nature of this peculiar phenomenon. 


\section{ACKNOWLEDGMENTS}

Stimulating discussions with P.F. Bortignon are greatly appreciated. We also thank M. Igashira and S. Mizuno for sending us their data on ${ }^{162}$ Dy. The authors are grateful to E.A. Olsen and J. Wikne for providing the excellent experimental conditions. This work is supported by the Norwegian Research Council (NFR).

[1] J.M. Blatt and V.F. Weisskopf, Theoretical Nuclear Physics (John Wiley \& Sons, New York, 1952).

[2] S.S. Dietrich and B.L. Berman, At. Data Nucl. Data Tables 38, 199 (1988).

[3] Yu. P. Popov, in Neutron Induced Reactions, Proceedings of the Europhysics Topical Conference, June 2125, 1982 Smolenice, Institute of Physics EPRC, Slovak Academy of Sciences, Bratislava 121 (1982).

[4] S.G. Kadmenskiǔ, V.P. Markushev, and V.I. Furman, Yad. Fiz. 37, 277 (1983) [Sov. J. Nucl. Phys. 37, 165 (1983)].

[5] J. Kopecky and M. Uhl, Phys. Rev. C 41, 1941 (1990).

[6] A. Bohr and B.R. Mottelson, Nuclear Structure (W.A. Benjamin, Inc., Reading, Massachusetts, 1975) Vol. II, p. 636 .

[7] J. Kopecky, M. Uhl, and R.E. Chrien, Phys. Rev. C 47, 312 (1993).

[8] M. Igashira, H. Kitazawa, M. Shimizu, H. Komano, and N. Yamamuro, Nucl. Phys. A457, 301 (1986).

[9] I. Bergqvist and N. Starfelt, Nucl. Phys. 39, 353 (1962).

[10] G.A. Bartholomew, I. Bergqvist, E.D. Earle, and A.J. Ferguson, Can. J. Phys. 48, 687 (1970).

[11] R.F. Barrett, K.H. Bray, B.J. Allen, and M.J. Kenny, Nucl. Phys. A278, 204 (1977).

[12] S. Joly, D.M. Drake, and L. Nilsson, Phys. Rev. C 20, 2072 (1979).

[13] M. Guttormsen, J. Rekstad, A. Henriquez, F. Ingebretsen, and T.F. Thorsteinsen, Phys. Rev. Lett. 52, 102 (1984).

[14] D. Bohle, A. Richter, W. Steffen, A.E.L. Dieperink, N. Lo Iudice, F. Palumbo, and O. Scholten, Phys. Lett. B 137, 27 (1984).

[15] L. Henden, L. Bergholt, M. Guttormsen, J. Rekstad, and T.S. Tveter, Nucl. Phys. A589, 249 (1995).

[16] T.S. Tveter, L. Bergholt, M. Guttormsen, E. Melby, and J. Rekstad, Phys. Rev. Lett. 77, 2404 (1996).
[17] A. Schiller, L. Bergholt, M. Guttormsen, E. Melby, J. Rekstad, and S. Siem, Nucl. Instrum. Methods Phys. Res. A 447, 498 (2000).

[18] E. Melby, L. Bergholt, M. Guttormsen, M. HjorthJensen, F. Ingebretsen, S. Messelt, J. Rekstad, A. Schiller, S. Siem, and S.W. Ødegård, Phys. Rev. Lett. 83, 3150 (1999).

[19] A. Schiller, A. Bjerve, M. Guttormsen, M. Hjorth-Jensen, F. Ingebretsen, E. Melby, S. Messelt, J. Rekstad, S. Siem, and S.W. Ødegård, nucl-ex/9909011.

[20] M. Guttormsen, A. Bjerve, M. Hjorth-Jensen, E. Melby, J. Rekstad, A. Schiller, S. Siem, and A. Belić, Phys. Rev. C 62, 024306 (2000).

[21] M. Guttormsen, A. Atac, G. Løvhøiden, S. Messelt, T. Ramsøy, J. Rekstad, T.F. Thorsteinsen, T.S. Tveter, and Z. Zelazny, Phys. Scr. T32, 54 (1990).

[22] D.M. Brink, Ph.D. thesis, Oxford University, 1955.

[23] P. Axel, Phys. Rev. 126, 671 (1962).

[24] A. Gilbert and A.G.W. Cameron, Can. J. Phys. 43, 1446 (1965).

[25] J. Kopecky and M. Uhl, in Proceedings of a Specialists' Meeting on Measurement, Calculation and Evaluation of Photon Production Data, Bologna, Italy 1994 (NEA/NSC/DOC(95)1) p. 119.

[26] S.F. Mughabghab, Neutron cross sections (Academic Press, New York, 1984), Vol. 1, part B.

[27] G.M. Gurevich, L.E. Lazareva, V.M. Mazur, S.Yu. Merkulov, G.V. Solodukhov, and V.A. Tyutin, Nucl. Phys. A351, 257 (1981).

[28] O.T. Grudzevich, Phys. Atom. Nucl. 62, 192 (1999).

[29] O.T. Grudzevich, Phys. Atom. Nucl. 63, 414 (2000).

[30] M. Guttormsen, M. Hjorth-Jensen, E. Melby, J. Rekstad, A. Schiller, and S. Siem, Phys. Rev. C 61, 067302 (2000).

[31] A.V. Ignatyuk, K.K. Istekov, and G.N. Smirenkin, Yad. Fiz. 29, 875 (1979) [Sov. J. Nucl. Phys. 29, 450 (1979)].

[32] A.V. Ignatyuk, J.L. Weil, S. Raman, and S. Kahane, Phys. Rev. C 47, 1504 (1993).

[33] E. Ramakrishnan et al. Phys. Lett. B 383, 252 (2000).

[34] W.E. Ormand, P.F. Bortignon, and R.A. Broglia, Phys. Rev. Lett. 77, 607 (1996).

[35] W.E. Ormand, P.F. Bortignon, R.A. Broglia, and A. Bracco, Nucl. Phys. A614, 217 (1997).

[36] Osman Yilmaz, Ahmet Gokalp, Serbulent Yildirim, and Sakir Ayik, Phys. Lett. B 472, 258 (2000).

[37] N. Dinh Dang, K. Eisenman, J. Seitz, and M. Thoennessen, Phys. Rec. C 61, 027302 (2000).

[38] F. Bečváŕ, P. Cejnar, J. Honzátko, K. Konečný, I. Tomandl, and R.E. Chrien, Phys. Rev. C 52, 1278 (1995).

[39] Satoshi Mizuno, Masayuki Igashira, and Koji Masuda, J. Nucl. Sci. Technol. 36, 493 (1999). 
TABLE I. The parameters used for calculation of $\gamma$-ray strength functions.

\begin{tabular}{|c|c|c|c|c|c|c|c|c|c|c|}
\hline Nucleus & $\begin{array}{c}E_{\mathrm{E} 1}^{(1)} \\
(\mathrm{MeV})\end{array}$ & $\begin{array}{c}\sigma_{\mathrm{E} 1}^{(1)} \\
(\mathrm{mb})\end{array}$ & $\begin{array}{c}\Gamma_{\mathrm{E} 1}^{(1)} \\
(\mathrm{MeV}) \\
\end{array}$ & $\begin{array}{c}E_{\mathrm{E} 1}^{(2)} \\
(\mathrm{MeV})\end{array}$ & $\begin{array}{c}\sigma_{\mathrm{E} 1}^{(2)} \\
(\mathrm{mb})\end{array}$ & $\begin{array}{c}\Gamma_{\mathrm{E} 1}^{(2)} \\
(\mathrm{MeV})\end{array}$ & $\begin{array}{c}E_{\mathrm{M} 1} \\
(\mathrm{MeV})\end{array}$ & $\begin{array}{c}\sigma_{\mathrm{M} 1} \\
(\mathrm{mb})\end{array}$ & $\begin{array}{c}\Gamma_{\mathrm{M} 1} \\
(\mathrm{MeV})\end{array}$ & $\begin{array}{c}\left\langle\Gamma_{\gamma}\right\rangle \\
(\mathrm{meV}) \\
\end{array}$ \\
\hline${ }^{161} \mathrm{Dy}$ & 12.13 & 210 & 2.6 & 15.8 & 250 & 5.05 & 7.66 & 1.60 & 4.0 & 108 \\
\hline${ }^{162}$ Dy & 12.13 & 210 & 2.6 & 15.8 & 250 & 5.05 & 7.65 & 1.49 & 4.0 & 113 \\
\hline${ }^{171} \mathrm{Yb}$ & 12.25 & 239 & 2.6 & 15.5 & 302 & 4.80 & 7.50 & 1.50 & 4.0 & 63 \\
\hline${ }^{172} \mathrm{Yb}$ & 12.25 & 239 & 2.6 & 15.5 & 302 & 4.80 & 7.50 & 1.76 & 4.0 & 75 \\
\hline
\end{tabular}

TABLE II. The parameters obtained from the fit.

\begin{tabular}{|c|c|c|c|c|c|}
\hline Nucleus & $\begin{array}{c}E_{\mathrm{py}} \\
(\mathrm{MeV})\end{array}$ & $\begin{array}{c}\sigma_{\mathrm{py}} \\
(\mathrm{mb})\end{array}$ & $\begin{array}{c}\Gamma_{\text {py }} \\
(\mathrm{MeV})\end{array}$ & $\begin{array}{c}T \\
(\mathrm{MeV})\end{array}$ & $K$ \\
\hline${ }^{161} \mathrm{Dy}$ & $2.69(4)$ & $0.49(5)$ & $1.37(22)$ & $0.29(11)$ & $1.34(11)$ \\
\hline${ }^{162} \mathrm{Dy}$ & $2.73(5)$ & $0.42(4)$ & $1.35(25)$ & $0.34(10)$ & $1.08(8)$ \\
\hline${ }^{171} \mathrm{Yb}$ & $3.35(6)$ & $0.65(7)$ & $0.97(16)$ & $0.34(3)$ & $1.22(10)$ \\
\hline${ }^{172} \mathrm{Yb}$ & $3.48(7)$ & $0.45(5)$ & $1.30(23)$ & $0.32(2)$ & $1.24(6)$ \\
\hline
\end{tabular}




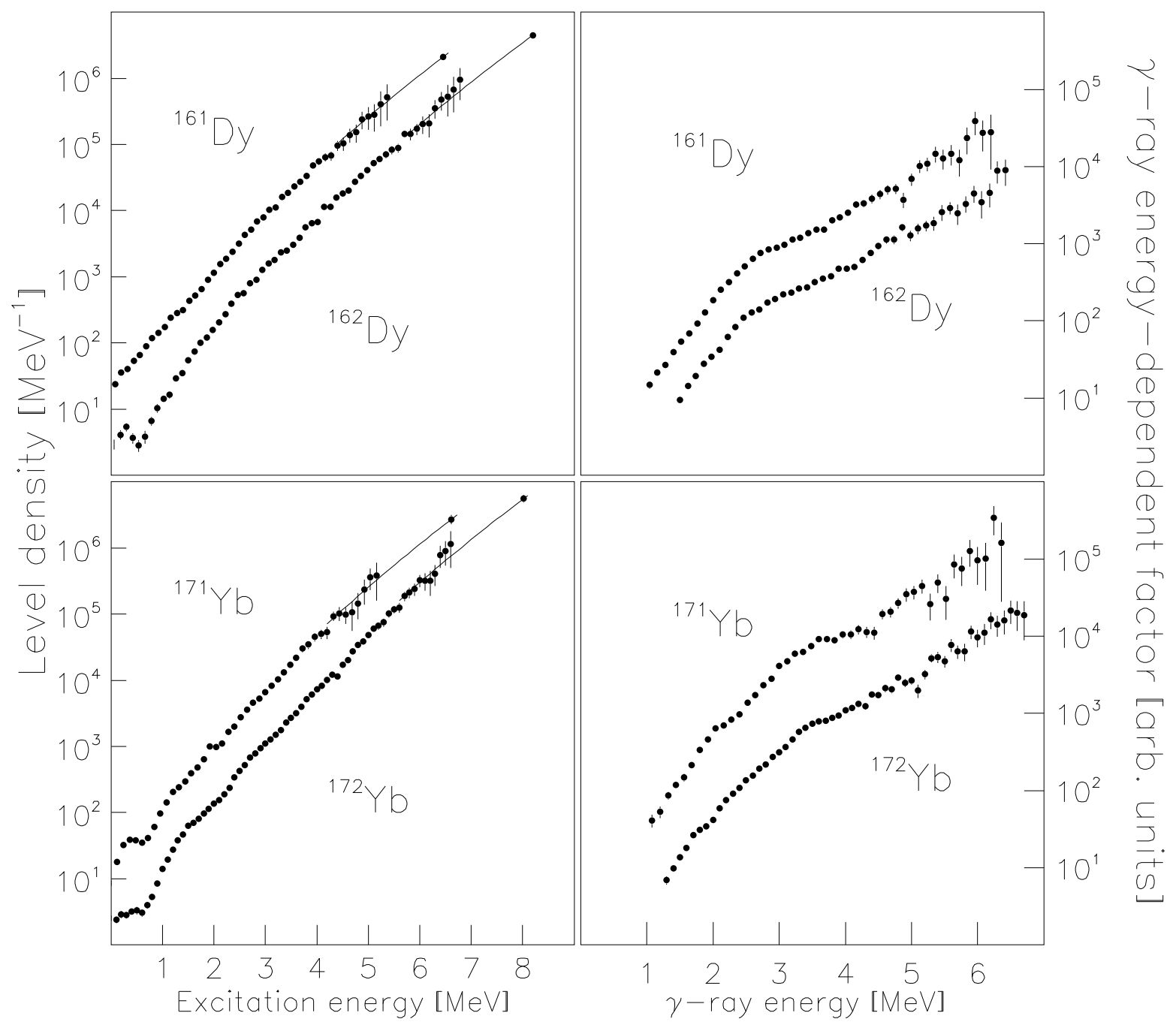

FIG. 1. The observed level density $\rho$ and the $\gamma$-ray energy dependent factor $F$ for ${ }^{161,162}$ Dy and ${ }^{171,172} \mathrm{Yb}$. 


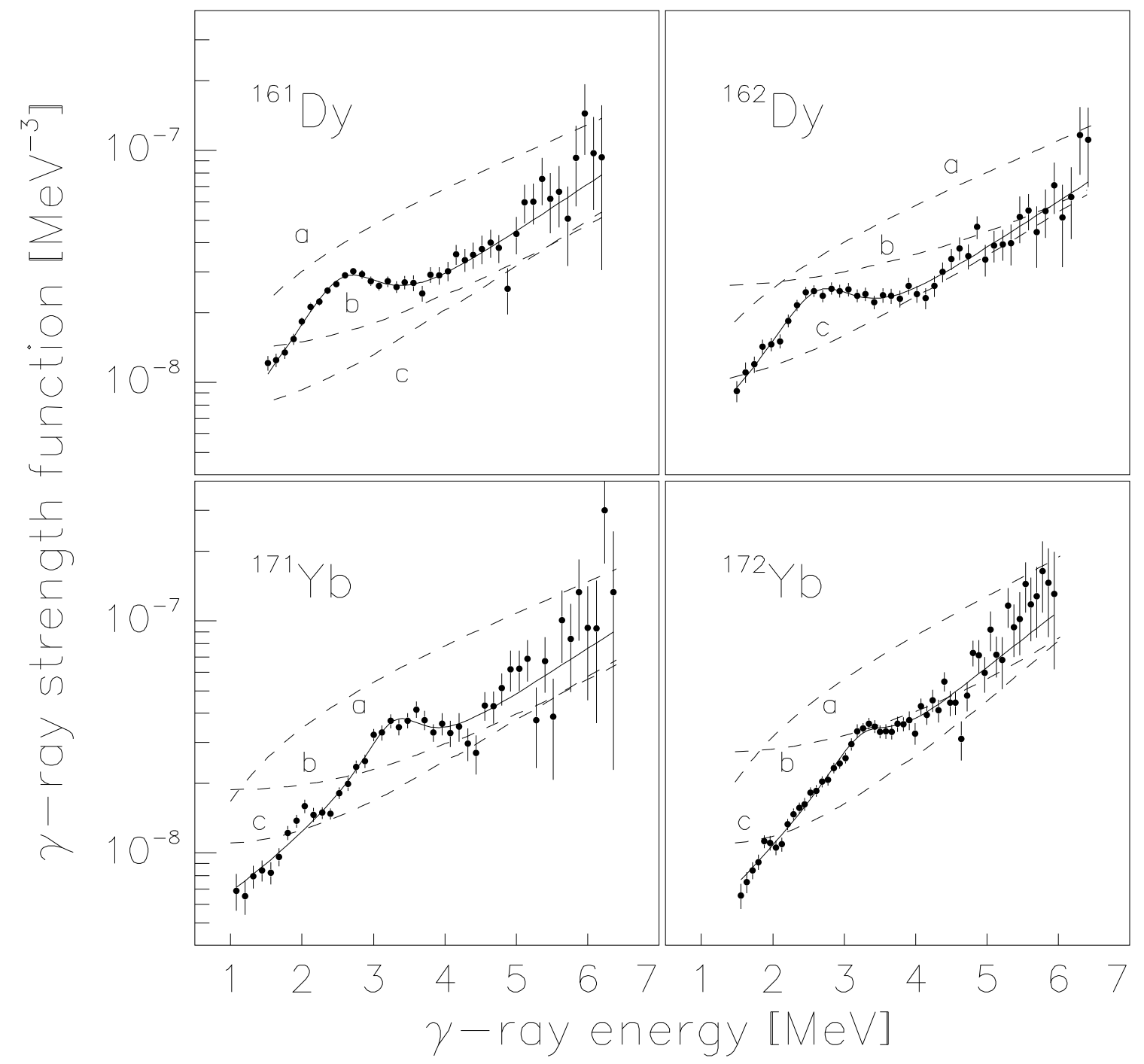

FIG. 2. The observed $\gamma$-ray strength functions (data points with error bars) for ${ }^{161,162}$ Dy and ${ }^{171,172}$ Yb. The dashed curves are calculations where a) denotes the Lorentzian GEDR model [Eq. (9)], b) the KMF model [Eq. (10)] plus a Weisskopf estimate for M1 transitions, and c) the KMF model [Eq. (10)] plus a Lorentzian GMDR model [Eq. (12)]. For b) and c), the temperature is given by $T=\sqrt{U / a}$. The solid curves are the KMF model with constant temperature [Eq. (10)] plus a Lorentzian GMDR model [Eq. (12)] plus a Lorentzian pygmy resonance model [Eq. (13)] (see text). 


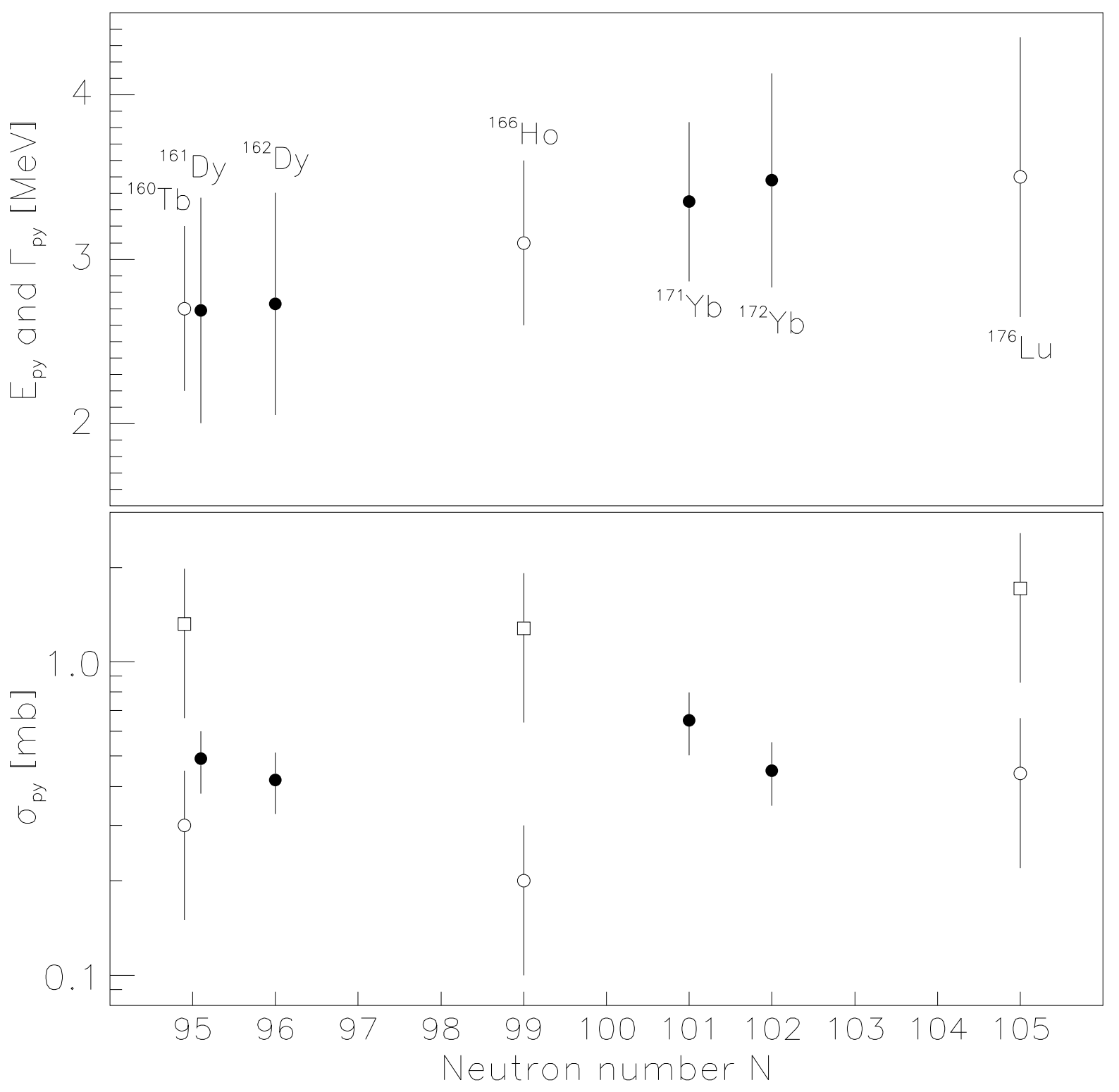

FIG. 3. Pygmy resonance parameters from the present $\left({ }^{3} \mathrm{He}, \alpha \gamma\right)$ reaction (filled circles) compared to those from the (n, $\gamma$ ) reaction [8] (open circles) as function of neutron number $N$. In the upper panel, the resonance energy $E_{\mathrm{py}}$ is displayed as data points and the width $\Gamma_{\text {py }}$ is given by the length of the lines through the data points. The cross-sections with error bars are shown in the lower panel. For the $\left({ }^{3} \mathrm{He}, \alpha \gamma\right)$ reaction, the quantity $\sigma_{\mathrm{py}}$ is plotted and assigned an additional systematic error of $20 \%$ from the normalization in Eq. (7). For the $(\mathrm{n}, \gamma)$ reaction, the quantities $\sigma_{\mathrm{py}}$ (open circles) and $k \sigma_{\mathrm{py}}$ (open squares) [8] are plotted. 


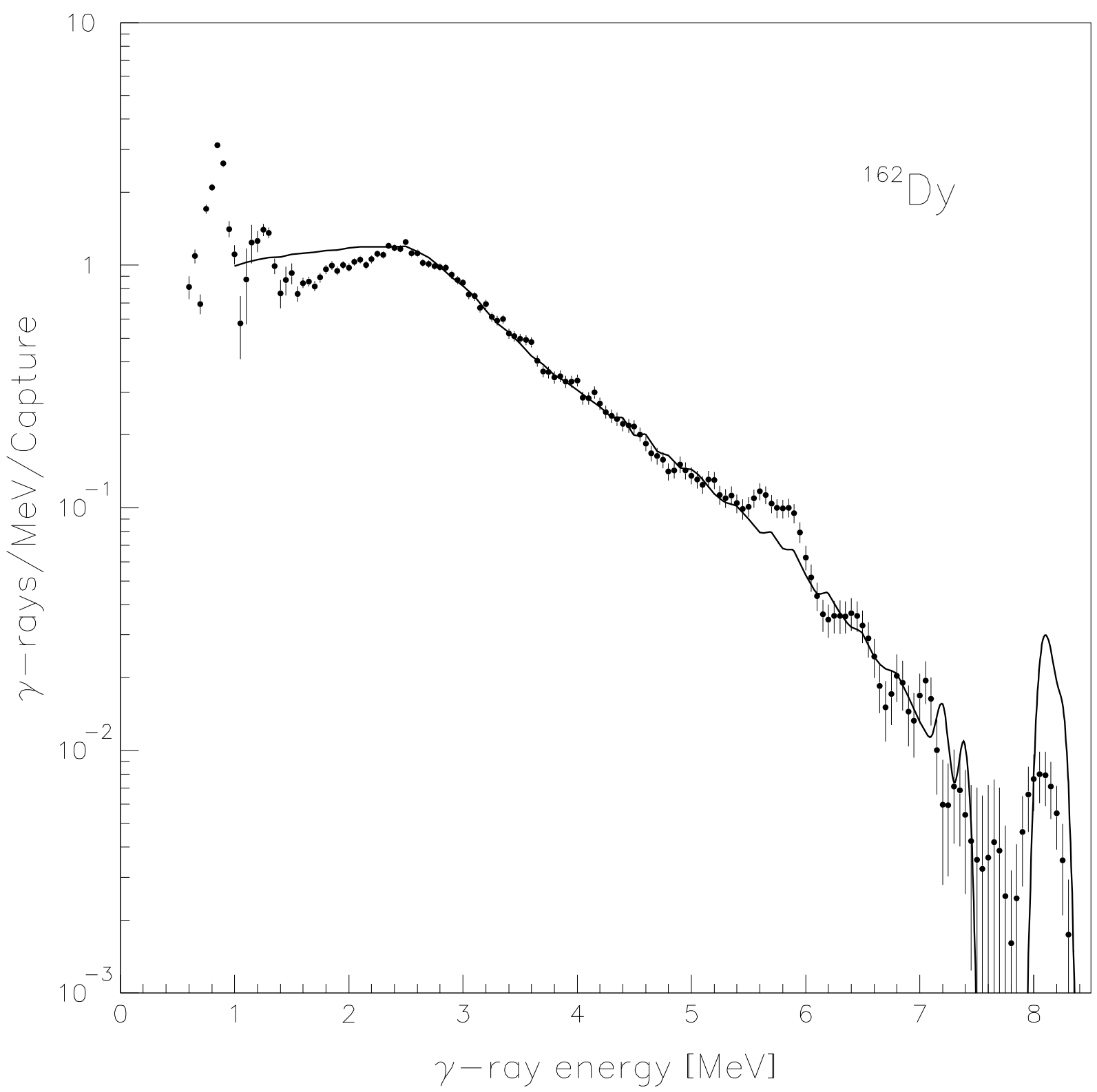

FIG. 4. The total $\gamma$-ray spectrum for ${ }^{162} \mathrm{Dy}$. The data points with error bars are taken from the ${ }^{161} \mathrm{Dy}(\mathrm{n}, \gamma){ }^{162} \mathrm{Dy}$ reaction $[39] \gamma$-ray strength function and the level density extracted from the present ${ }^{163} \mathrm{Dy}\left({ }^{3} \mathrm{He}, \alpha \gamma\right){ }^{162} \mathrm{Dy}$ data. The calculation is performed by averaging over $100 \mathrm{keV}$ intervals. 